\title{
Maternal Psychological Distress, Education, Household Income, and Congenital Heart Defects: A Prospective Cohort Study from The Japan Environment and Children's Study Running Title Maternal Psychological Distress and Congenital Heart Defects
}

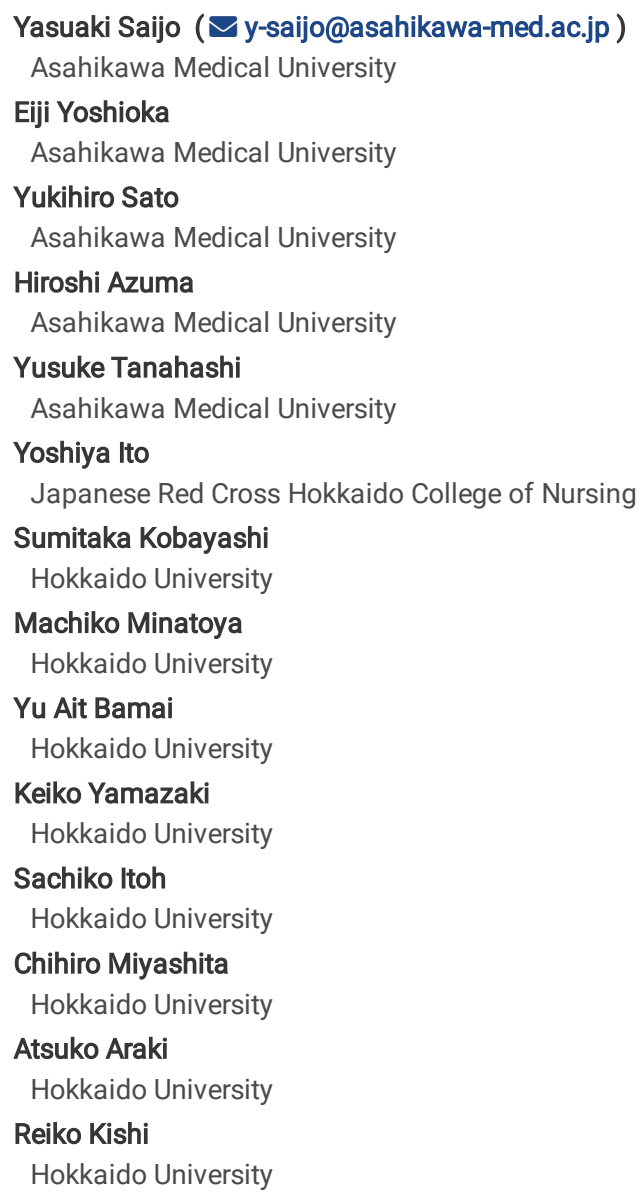

\section{Research Article}

Keywords: congenital heart defects, psychological distress, education, birth cohort

Posted Date: March 24th, 2021

DOI: https://doi.org/10.21203/rs.3.rs-289676/v1

License: (9) (i) This work is licensed under a Creative Commons Attribution 4.0 International License. Read Full License

Version of Record: A version of this preprint was published at BMC Pregnancy and Childbirth on August 7th, 2021. See the published version at https://doi.org/10.1186/s12884-021-04001-2. 


\section{Abstract}

Background: The influence of maternal psychological distress on infant congenital heart defects (CHDs) has not been thoroughly investigated. Furthermore, there have been no reports on the combined effect of maternal psychological distress and socioeconomic status on infant CHDs. This study aimed to examine whether maternal psychological distress, socioeconomic status, and their combinations were associated with CHD.

Methods: We conducted a prospective cohort study using data from the Japan Environment and Children's Study, which recruited pregnant women between 2011 and 2014. Maternal psychological distress was evaluated using the Kessler Psychological Distress Scale in the first trimester, while maternal education and household income were evaluated in the second and third trimesters. The outcome of infant CHD was determined using the medical records at 1 month of age and/or at birth. Crude and adjusted logistic regression analyses were performed to evaluate the association between maternal psychological distress and education and household income on infant CHD.

Results: A total of 93,643 pairs of mothers and infants were analyzed, with $1.1 \%$ of infants having CHDs. Maternal psychological distress had a significantly higher odds ratio in the crude analysis but not in the adjusted analysis, while maternal education and household income were statistically insignificant. In the analysis of the combination variable of lowest education and psychological distress, the trend for $\mathrm{p}$ was statistically significant in the crude and adjusted analyses.

Conclusions: The combination of maternal psychological distress and lower education may be a possible indicator of infant CHD.

\section{Background}

Congenital heart defects (CHDs) are the most common non-chromosomal congenital disorders [1] and develop in 0.8 to $1.2 \%$ of newborns [2-4]. Although CHD-related mortality has dramatically decreased due to the significant improvements in diagnostics, percutaneous interventions, surgery, and medication, enabling most children to reach adulthood [3], it remains the leading cause of mortality from congenital anomalies and results in a heavy global disease burden $[4,5]$.

A meta-analysis published in 2014 reported that lower maternal socioeconomic status was slightly associated with increased risk of infant CHDs, while lower maternal education and household income had significantly higher relative risks of 1.11 and 1.05 for infant CHDs, respectively [6]. Furthermore, recent casecontrol studies have also reported a significant risk of maternal lower education for CHD [7, 8]. A lower socioeconomic status is associated with common mental disorders [9, 10], and the association between anti-depressant use during pregnancy and CHD has been reported in a meta-analysis [11]. Concerning actual depressive symptoms, a case-control study in China reported that depressive symptoms during pregnancy were related to a significantly increased odds ratio (OR) for infant CHDs [12]. However, the diagnosis of depression in a UK cohort and depressive symptoms in a Northern Ireland case-control study was not related to infant CHDs $[8,13]$.

Thus, whether maternal psychological distress, including depressive symptoms, is a risk factor for CHD has not been fully investigated, and there have been no reports on the combined effects of maternal education, income, and maternal psychological distress on CHD. Further, to our knowledge, there have been no Japanese reports investigating maternal education, income, and maternal psychological distress on CHD risk after adjusting for confounders. This study aimed to examine whether maternal psychological stress, education, household income, and their combinations affected infant CHD using the birth cohort data from the Japan Environment and Children's Study (JECS) [14, 15].

\section{Methods}

\section{Participants}

The JECS is a nationwide Japanese prospective birth cohort study aiming to identify the environmental factors affecting children's health and development $[14,15]$. To cover all the geographical areas of Japan, pregnant women were recruited from 15 Regional Centers (Hokkaido, Miyagi, Fukushima, Chiba, Kanagawa, Koshin, Toyama, Aichi, Kyoto, Osaka, Hyogo, Tottori, Kochi, Fukuoka, and South Kyushu/Okinawa). Baseline recruitment was performed in collaboration with local governments and healthcare providers to maximize representativeness. The children were then followed up until 13 years from birth.

Between January 2011 and March 2014, 103,060 pregnant women in the early stages of pregnancy were recruited. Excluding the pregnancies in the same woman, the study involved 97,413 unique pregnancies. In our study, we excluded 3,561 pregnancies with a birth status of miscarriage or stillbirth. Of the remaining 93,852 births, which included only the first infant among those with multiple births, those diagnosed with chromosomal abnormalities ( $\mathrm{n}=209$ ), based on their medical records at birth and 1 month, were excluded. Thus, the final number of participants was 93,643 infants (Figure 1). Specifically, we used the jecs-ta-20190930 dataset from the JECS, registered in the University Hospital Medical Information Network (UMIN) 000030786 (UMIN Clinical Trials Registry, 15/01/2018).

\section{Ethics statement}

The JECS protocol was approved by the Institutional Review Board on Epidemiological Studies of the Ministry of the Environment and by the ethics committees of all participating institutions. This was also conducted in accordance with the Declaration of Helsinki and other nationally valid regulations. Written informed consent was obtained from all participants.

\section{Outcomes}


Infant CHD diagnosed in the medical records at 1 month of age and/or at birth was defined as the outcome. In the sensitivity analyses, the medical records were checked if the caregivers answered positively to the diagnosis of CHD after birth among the infants' siblings in the 2-year questionnaire. If CHDs were confirmed in the medical records, we defined them as 2-year CHD-positive in the sensitivity analysis.

\section{Maternal and education, household income, and psychological distress}

During pregnancy, questionnaires were distributed to the enrolled mothers during the first $(\mathrm{T} 1$; if the participation was delayed, it was distributed during the second/third trimester) and second/third trimesters (T2). The latter included questions about the mothers' educational attainment, categorized as $\leq 9$ years (EDC1: junior high school), 10 to $\leq 12$ years (EDC2: high school), 13-15 years (EDC3: technical junior college, technical/vocational college, or associate degree), or $\geq 16$ years (EDC4: bachelor's degree or postgraduate degree). The T2 questionnaire also included questions on household income, categorized as $\leq 199,200-399,400-599,600-799,800-999$, and $\geq 1000$ thousand yen. Meanwhile, maternal psychological distress was assessed using the Japanese version of the Kessler 6-Item Psychological Distress Scale (K6) in the T1 questionnaire [16, 17], with a K6 score of >13 points indicating positive maternal psychological distress $[18,19]$.

\section{The other independent variables}

Based on previous studies [7-10, 12,13], the following were selected as covariates: maternal age at delivery, pregnancy body mass index (BMI), paternal education, marital status, mother's alcohol habit, mother and father's smoking habits, parity, infant sex, plurality, fertility treatment, hypertensive disorder during pregnancy, thyroid diseases during pregnancy, diabetes mellitus/gestational diabetes during pregnancy, folic acid supplementation at early pregnancy, anti-depressant use at early pregnancy, and mother's CHDs.

The T1 questionnaire included questions regarding the mother's birthday, marital status, smoking habit (along with the father's), folic acid supplementation, anti-depressant use, and history of CHDs. Marital status was classified as married or unmarried, including divorced or bereaved. Smoking habit was categorized as never smoked/quitting smoking before pregnancy or quitting smoking/continued smoking during pregnancy. Folic acid supplementation and anti-depressant use were defined as positive if these were taken between pregnancy perception and 12 weeks of gestation. Lastly, if the mothers responded positively to the query about a previous CHD diagnosis, they were considered positive for CHDs.

On the other hand, the T2 questionnaire inquired about the mothers' drinking habits. Nondrinkers included those with no history of alcohol intake and those quitting before pregnancy, while drinkers including those currently drinking or quitting during pregnancy.

The following information was also collected from the medical records: infant birth date, plurality, parity, mode of pregnancy (spontaneous, ovulation induction through medication, or artificial insemination/in vitro fertilization), hypertension (hypertension before or during pregnancy), thyroid disease, diabetes mellitus (diabetes mellitus before or during pregnancy), and height and pre-pregnancy weight, from which the BMI was calculated. The mother's age at infant birth was calculated using her and the infant's birth dates.

\section{Statistical analysis}

Fisher's exact test was used to analyze the associations between the outcome of infant CHD and maternal age, pre-pregnancy BMI, maternal education, father education, household income, marital status, mother drinking habit, mother smoking, father smoking, plurality, infant sex, mode of pregnancy, hypertensive disorder, thyroid diseases, diabetes, folic acid supplementation, anti-depressant, mother CHDs, and maternal psychological distress.

For participants with missing data (1.9\%), the information was replaced using multiple imputations (25 imputed datasets) based on the assumption that data were missing at random. The imputation model included all the variables analyzed in Fisher's exact test and the K6 raw score, dichotomized to K6 $\geq 13$ or not. Using the imputed datasets, the crude OR of each variable for infant CHD was calculated.

Next, we conducted multivariable logistic regression analyses to estimate the ORs for infant CHD with $95 \%$ confidence intervals (95\% Cls). First, psychological distress, maternal education, and household income were introduced separately (crude model). In model 1, the three variables were introduced, along with age, pre-pregnancy BMI, father education, marital status, mother drinking habit, mother smoking, paternal smoking, plurality, infant sex, mode of pregnancy, hypertensive disorder, thyroid diseases, diabetes, and folic acid supplementation. Despite the varying reports on anti-depressants being possible mediators of depression [8, 20-23], model 2 was constructed using all the model 1 variables and anti-depressant use.

Then, we constructed the combination variable of the mothers' lowest education (EDC1) and psychological distress. Lower household income was not included as a combination variable because it had no significant protective OR. We then analyzed the crude and adjusted OR of the combination variable, and the trend $P$ values were calculated using it as an integer variable.

As mentioned above, 2-year CHD data was available, but this was restricted to the participants who had answered the 2-year questionnaire ( $\mathrm{N}=80,468)$, which may have been biased towards the participants with higher education, higher household income, and lower psychological distress tended to respond to the 2year questionnaire (Additional file 1). Therefore, in the first sensitivity analysis, 2-year CHD positivity was added to the original CHDs positive outcome, but in the second sensitivity analysis, it was deleted from the original outcome negative.

Two-sided P-values of $<0.05$ were considered statistically significant. All analyses were conducted using Stata statistical software version 16.0 for Windows (StataCorp, College Station, TX, USA).

\section{Results}


CHD had a prevalence of $1.1 \%$ (Table 1). Among maternal education, household income, and psychological distress, only psychological distress had a statistically significant relationship with infant CHDs in the pairwise deletion analyses (Additional file 2). 


\begin{tabular}{|c|c|c|}
\hline & $\mathbf{N}$ & $\%$ \\
\hline \multicolumn{3}{|l|}{ Mother's age at delivery } \\
\hline-24 & 9,378 & 10.0 \\
\hline $25-29$ & 25,755 & 27.5 \\
\hline $30-34$ & 33,022 & 35.3 \\
\hline $35-39$ & 21,159 & 22.6 \\
\hline $40-$ & 4,318 & 4.6 \\
\hline Missing & 11 & 0.0 \\
\hline \multicolumn{3}{|l|}{ Pre-pregnancy BMI } \\
\hline-18.4 & 14,652 & 15.7 \\
\hline $18.5-24.9$ & 68,683 & 73.4 \\
\hline $25-$ & 10,180 & 10.9 \\
\hline Missing & 128 & 0.1 \\
\hline \multicolumn{3}{|l|}{ Mother's education } \\
\hline EDC1 & 4,389 & 4.7 \\
\hline EDC2 & 28,664 & 30.6 \\
\hline EDC3 & 38,499 & 41.1 \\
\hline EDC4 & 19,910 & 21.3 \\
\hline Missing & 2,181 & 2.3 \\
\hline \multicolumn{3}{|l|}{ Father's education } \\
\hline EDC1 & 6,546 & 7.0 \\
\hline EDC2 & 33,325 & 35.6 \\
\hline EDC3 & 20,512 & 21.9 \\
\hline EDC4 & 30,481 & 32.6 \\
\hline Missing & 2,779 & 3.0 \\
\hline \multicolumn{3}{|c|}{ Household income (10 thousand yen/year) } \\
\hline-199 & 4,820 & 5.2 \\
\hline $200-399$ & 29,378 & 31.4 \\
\hline $400-599$ & 28,177 & 30.1 \\
\hline $600-799$ & 13,642 & 14.6 \\
\hline 800-999 & 5,677 & 6.1 \\
\hline $1000-$ & 3,652 & 3.9 \\
\hline Missing & 8,297 & 8.9 \\
\hline \multicolumn{3}{|l|}{ Marital status } \\
\hline Married & 87,804 & 93.8 \\
\hline Unmarried, divorced or bereavement & 4,189 & 4.5 \\
\hline Missing & 1,650 & 1.8 \\
\hline \multicolumn{3}{|l|}{ Mother's alcohol intake } \\
\hline Non-drinker & 45,632 & 48.7 \\
\hline Drinker during early pregnancy & 45,604 & 48.7 \\
\hline Missing & 2,407 & 2.6 \\
\hline
\end{tabular}


Mother's smoking status

Non-, ex-smoker

74,878

80.0

Smoker during pregnancy

16,840

18.0

Missing

1,925

2.1

Father's smoking status

Non-, ex-smoker

45,760

48.9

Smoker during pregnancy

44,594

47.6

Missing

3,289

3.5

Plurality

Singleton

92,738

99.0

Multiplet

905

1.0

Parity

0

38,925

41.6

1

$\geq 2$

34,151

36.5

18,183

19.4

Missing

2,384

2.6

Infant's sex

Boy

45,587

48.7

Girl

48,038

51.3

Missing

18

0.0

Fertility treatment

Spontaneous

86,724

92.6

Ovulation induction through medication

2,598

2.8

Artificial insemination or in vitro Fertilization

3,816

4.1

Missing

505

0.5

Hypertensive disorder during pregnancy

No

88,703

94.7

Yes

3,397

3.6

Missing

1,543

1.7

Thyroid diseases during pregnancy

No

90,362

96.5

Yes

1,244

1.3

Missing

2,037

2.2

Diabetes mellitus during pregnancy/gestational diabetes

No

89,160

95.2

Yes

2,940

3.1

Missing

1,543

1.7

Folic acid supplementation at early pregnancy

No

66,290

70.8

Yes

25,585

27.3

Missing

1,768

1.9

EDC1: junior high school, EDC2: high school, EDC3: technical junior college, technical/vocational college, or EDC4: associate degree bachelor's degree or postgraduate degree. 


\begin{tabular}{|c|c|c|}
\hline & $\mathbf{N}$ & $\%$ \\
\hline \multicolumn{3}{|c|}{ Anti-depressant use } \\
\hline No & 91,651 & 97.9 \\
\hline Yes & 224 & 0.2 \\
\hline Missing & 1,768 & 1.9 \\
\hline \multicolumn{3}{|c|}{ Congenital heart diseases in the mother } \\
\hline No & 92,119 & 98.4 \\
\hline Yes & 289 & 0.3 \\
\hline Missing & 1,235 & 1.3 \\
\hline \multicolumn{3}{|c|}{ Psychological distress in the mother } \\
\hline No & 88,072 & 94.1 \\
\hline Yes & 3,231 & 3.5 \\
\hline Missing & 2,340 & 2.5 \\
\hline \multicolumn{3}{|c|}{ CHD (outcome) in the siblings } \\
\hline Negative & 92,641 & 98.9 \\
\hline Positive & 1,002 & 1.1 \\
\hline
\end{tabular}

After multiple imputations in the crude logistic regressions, psychological distress had a significantly higher OR (OR 1.39; 95\% Cl, 1.03-1.87), while maternal education and household income were statistically insignificant. However, in models 2 and 3 , the significance of psychological distress became dismissible (Model 1: OR 1.32; 95\% Cl, 0.98-1.79; Model2: OR 1.31; 95\% Cl, 0.97-1.77) (Table 2). 
Table 2

Crude and adjusted ORs of maternal education, psychological distress, and household income (Multiple imputation, $\mathrm{N}=93,643$ )

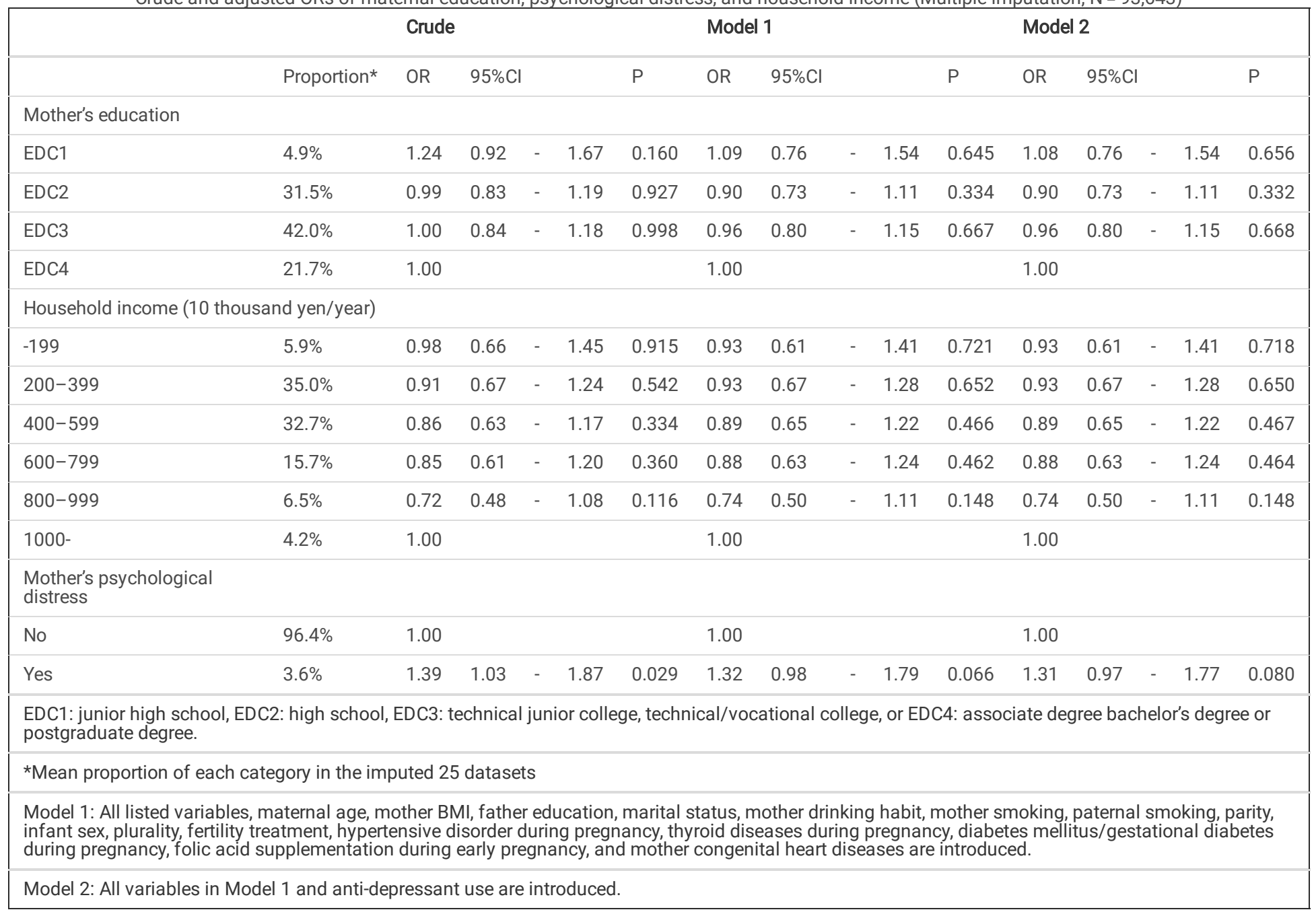

In the analysis of the combination variable of maternal psychological distress and lowest education (EDC1), the first and second combinations had no statistical significance, but the trend for $p$ was statistically significant (crude: $p=0.011$, Model $1 ; P=0.043$ ). However, its significance diminished in Model 2 ( $p$ $=0.050$ ) (Table 3, ORs of the combination of EDC1 and psychological distress are shown in Supplemental Table 3). 
Table 3

Adjusted ORs of number of maternal education and psychological distress for CHDs (Multiple imputation, 93,643)

\begin{tabular}{|c|c|c|c|c|c|c|c|c|c|c|c|c|c|c|c|c|c|c|c|}
\hline & \multirow[b]{2}{*}{ Proportion* } & \multicolumn{6}{|c|}{ Crude } & \multicolumn{6}{|c|}{ Model 1} & \multicolumn{6}{|c|}{ Model 2} \\
\hline & & OR & $95 \% \mathrm{Cl}$ & & & $P$ & $\begin{array}{l}P \text { for } \\
\text { trend }\end{array}$ & OR & $95 \% \mathrm{Cl}$ & & & $\mathrm{P}$ & $\begin{array}{l}P \text { for } \\
\text { trend }\end{array}$ & OR & $95 \% \mathrm{Cl}$ & & & $\mathrm{P}$ & $\begin{array}{l}\mathrm{P} 1 \\
\text { tre }\end{array}$ \\
\hline \multicolumn{20}{|c|}{$\begin{array}{l}\text { Number } \\
\text { of } \\
\text { positive }\end{array}$} \\
\hline 0 & $92.0 \%$ & 1.00 & & & & & 0.011 & 1.00 & & & & & 0.043 & 1.00 & & & & & 0.1 \\
\hline 1 & $7.6 \%$ & 1.25 & 1.00 & - & 1.55 & 0.052 & & 1.20 & 0.95 & - & 1.52 & 0.123 & & 1.20 & 0.95 & - & 1.51 & 0.135 & \\
\hline 2 & $0.4 \%$ & 2.02 & 0.97 & - & 4.24 & 0.062 & & 1.87 & 0.88 & - & 3.99 & 0.103 & & 1.83 & 0.86 & - & 3.91 & 0.117 & \\
\hline
\end{tabular}

*Mean proportion of each category in the imputed 25 datasets

**Number of mother junior high school or psychological distress positive

Model 1: All listed variables, maternal age, mother BMI, household income father education, marital status, mother drinking habit, mother smoking, paternal smoking, parity, infant sex, plurality, fertility treatment, hypertensive disorder during pregnancy, thyroid diseases during pregnancy, diabetes mellitus/gestatior diabetes during pregnancy, folic acid supplementation during early pregnancy, and mother congenital heart diseases are introduced.

Model 2: All variables in Model 1 and anti-depressant use are introduced.

P for trend: The number of positives (0-2) was introduced into the model.

* Only the first infants of multiple births are included.

In the first sensitivity analysis where the 2-year CHDs positive were added to the original outcome positive, psychological distress had a significantly higher OR only in the crude analysis (OR 1.30; 95\% Cl, 1.00-1.69) (Supplemental Table 4). A similar result was observed in the second sensitivity analysis where the 2year CHDs positive were deleted from the original outcome negative (OR 1.39; 95\% Cl, 1.03-1.87) (Supplemental Table 5). Regarding the combination variable of psychological distress and lowest education, the significant trend for p disappeared in the first sensitivity analysis (Supplemental Table 6 ) but was still significant in crude and model 1 in the second sensitivity analysis (Supplemental Table 7).

\section{Discussions}

In this prospective birth cohort study, the crude analysis showed that only maternal psychological distress had a significantly higher OR for infant CHDs in the crude analysis, while lower education in mothers and lower household income were unexpectedly insignificant. Further, the combination variable of maternal psychological distress and lowest education had significant trend p values in the crude analysis and Model 1 but not in Model 2.

As previously mentioned, the meta-analysis published in 2014 reported that lower maternal education and household income had significantly higher relative risks for infant CHDs at 1.11 and 1.05, respectively [6]. While the relative risk of household income was significant, its effect size was negligible relative to maternal education. The protective results of higher household income in the present study may be due to the minimal effect size. Furthermore, household income did not necessarily reflect the lifetime socioeconomic status for relatively young women. Education was related to favorable dietary intake patterns among pregnant Japanese women, but the household income was not [24].

In our current study, maternal education had no significant result with the point estimation of crude OR 1.24. Although its OR was higher than household income in the meta-analysis [6], the significance of the relatively low effect size may not be detected due to the lack of statistical power. Moreover, socioeconomic effects on CHDs in developed countries were smaller compared to developing countries [6], and it has been reported that socioeconomic differences affected mortality, morbidity, and risk factors in Japan to a lesser degree than in the US or Europe [25].

A Chinese case-control study found that prenatal depressive symptoms had significantly increased OR (1.94) for CHD. However, the symptoms were evaluated using the Zung Self-Rating Depression Scale after birth, leading to recall bias [12]. Meanwhile, in the UK cohort, diagnosis of depression without antidepressant use during the first trimester had no significant OR (1.10). Similarly, a case-control study conducted in Northern Ireland reported that having symptoms of "feeling down, depressed or hopeless" within the past month at 10-12 weeks of gestation had no significant OR $(1.20)$ [8, 13]. In the present study, psychological distress had a significant OR on crude analysis, but this became negligible in the adjusted models. The K6 scores in our study could not be directly compared with the previous studies' because this test evaluates psychological distress and does not focus on depression only [17-19], and the evaluation of depression varied across the studies. However, psychological distress, if present, seemed to have a minor effect on infant CHD.

The combination variable of maternal psychological distress and lowest education (EDC1) had significant trend P values in the crude analysis and Model 1 , but both had no statistically significant ORs $(2.02,95 \%$ confidence interval: $0.97-4.24)$ in the crude analysis. Because the effect size of the crude model was relatively large, the combination may identify high-risk pregnancies for infant $\mathrm{CHD}$. The significance of trend $\mathrm{p}$ disappeared in Model 2 , and the mediation effect of anti-depressants may influence the significance of dilution, although it seemed to be weak [8].

There were doctor-diagnosed child CHDs beyond 2 years among the participants who answered the 2-year age questionnaire, who tended to have a higher socioeconomic status and a lower prevalence of psychological distress [26]. Therefore, to negate this possible bias, we performed two sensitivity analyses. The first analysis included the 2-year CHDs positive and the original CHDs positive outcome, which was almost slightly diluted, as expected. On the other

Page $9 / 12$ 
hand, in the second sensitivity analysis, several p values decreased slightly when the 2-year CHDs positive were deleted from the outcome negative. In the main analysis, the relationships were mostly biased to null because the diagnosis of CHDs seemed to result in the nondifferential misclassification of the binary outcomes [27]. Therefore, we believe that the crude effects of psychological distress and the combination variable are small but significant.

There were several limitations to our present study. First, we did not have adequate statistical power to investigate the weak relationships. For instance, assuming that the non-exposure groups had an average CHD incidence (1.07\%), the statistical power to obtain ORs of 1.3 for ECD1 (reference: ECD4) and maternal psychological distress would be $46 \%$ and $43 \%$, respectively. Second, our results may not be generalized to other countries due to differences in the educational systems, rates of entering higher educational institutions, and the prevalence of maternal psychological distress. Third, prenatal diagnosis of CHD may affect maternal psychological distress [28]. However, its effect during the first trimester might be limited because the fetal CHDs were mainly diagnosed after 18 to 20 gestational weeks [29], and $80 \%$ of the T1 questionnaires were submitted within 20 gestational weeks.

\section{Conclusions}

The crude analysis in this prospective birth cohort study showed that maternal psychological distress in the first trimester was related to infant CHDs. Meanwhile, the multivariate analysis showed the maternal psychological distress and lowest education were also related. The combination of maternal psychological distress and lowest education may be a possible indicator of infant CHD.

\section{Abbreviations}

CHD, congenital heart defects; JECS, Japan Environment and Children's Study; EDC, education; K6, 6-Item Psychological Distress Scale; OR, odds ratio; Cl, confidence interval

\section{Declarations}

\section{Ethics approval and consent to participate}

The JECS protocol was approved by the Institutional Review Board on Epidemiological Studies of the Ministry of the Environment and by the ethics committees of all the participating institutions. The JECS was conducted in accordance with the Declaration of Helsinki and other nationally valid regulations. Written informed consent was obtained from all participating mothers and fathers.

\section{Consent for publication}

Not applicable.

\section{Availability of data and materials}

Data availability:

Data are unsuitable for public deposition because of the ethical restrictions and legal framework of Japan. Specifically, the Act on the Protection of Personal Information (Act No. 57 of May 30, 2003, amended on September 9, 2015) prohibits the public deposition of data containing personal information. The Ethical Guidelines for Medical and Health Research Involving Human Subjects, enforced by the Japan Ministry of Education, Culture, Sports, Science and Technology and the Ministry of Health, Labour and Welfare, also restrict the open sharing of epidemiologic data. All inquiries about access to data were sent to: jecsen@nies.go.jp, handled by Dr. Shoji F. Nakayama of the JECS Programme Office, National Institute for Environmental Studies.

\section{Competing interests}

The authors declare that they have no competing interests.

\section{Funding}

The Japan Environment and Children's Study was funded by the Ministry of Environment, Japan. The findings and conclusions of this study are solely the responsibility of the authors and do not represent the official views of the abovementioned government agency.

\section{Authors' contributions}

The JECS group collected the data and obtained the funding. YaS was a major contributor in writing the manuscript, and analyzing and interpreting the data. All the other co-authors (EY, YuS, HA, YT, YI, SK, MM, YAB, KY, SI, CM, AA, and RK) made substantive contributions to the conception of the study, interpretation of the results, and critically reviewing the draft. All authors read and approved the final draft of the manuscript.

\section{Acknowledgements}

We would like to express our gratitude to all the participants of the JECS and all the staff members involved in data collection.

Members of the JECS Group as of 2020: Michihiro Kamijima (principal investigator, Nagoya City University, Nagoya, Japan), Shin Yamazaki (National Institute for Environmental Studies, Tsukuba, Japan), Yukihiro Ohya (National Center for Child Health and Development, Tokyo, Japan), Reiko Kishi (Hokkaido 
University, Sapporo, Japan), Nobuo Yaegashi (Tohoku University, Sendai, Japan), Koichi Hashimoto (Fukushima Medical University, Fukushima, Japan), Chisato Mori (Chiba University, Chiba, Japan), Shuichi Ito (Yokohama City University, Yokohama, Japan), Zentaro Yamagata (University of Yamanashi, Chuo, Japan), Hidekuni Inadera (University of Toyama, Toyama, Japan), Takeo Nakayama (Kyoto University, Kyoto, Japan), Hiroyasu Iso (Osaka University, Suita, Japan), Masayuki Shima (Hyogo College of Medicine, Nishinomiya, Japan), Youichi Kurozawa (Tottori University, Yonago, Japan), Narufumi Suganuma (Kochi University, Nankoku, Japan), Koichi Kusuhara (University of Occupational and Environmental Health, Kitakyushu, Japan), and Takahiko Katoh (Kumamoto University, Kumamoto, Japan).

\section{References}

1. Dolk H, Loane M, Garne E. The prevalence of congenital anomalies in Europe. Adv Exp Med Biol 2010; 686:349-64.

2. Liu Y, Chen S, Zühlke L, Black GC, Choy MK, Li N, et al. Global birth prevalence of congenital heart defects 1970-2017: updated systematic review and meta-analysis of 260 studies. Int J Epidemiol 2019; 48(2):455-63.

3. Bouma BJ, Mulder BJ. Changing Landscape of Congenital Heart Disease. Circ Res 2017; 120(6):908-22.

4. Wu W, He J, Shao X. Incidence and mortality trend of congenital heart disease at the global, regional, and national level, 1990-2017. Medicine (Baltimore) 2020; 99(23):e20593.

5. Collaborators. GCoD. Global, regional, and national age-sex-specific mortality for 282 causes of death in 195 countries and territories, 1980-2017: a systematic analysis for the Global Burden of Disease Study 2017. Lancet 2018; 392(10159):1736-88.

6. Yu D, Feng Y, Yang L, Da M, Fan C, Wang S, et al. Maternal socioeconomic status and the risk of congenital heart defects in offspring: a meta-analysis of 33 studies. PLoS One 2014; 9(10):e111056.

7. Arjmandnia M, Besharati M, Rezvan S. Studying the determinant factors leading to congenital heart disease in newborns. J Educ Health Promot 2018; 7:53.

8. Dolk H, McCullough N, Callaghan S, Casey F, Craig B, Given J, et al. Risk factors for congenital heart disease: The Baby Hearts Study, a population-based case-control study. PLoS One 2020; 15(2):e0227908.

9. Fryers T, Melzer D, Jenkins R. Social inequalities and the common mental disorders: a systematic review of the evidence. Soc Psychiatry Psychiatr Epidemiol 2003; 38(5):229-37.

10. Jokela M, Batty GD, Vahtera J, Elovainio M, Kivimäki M. Socioeconomic inequalities in common mental disorders and psychotherapy treatment in the UK between 1991 and 2009. Br J Psychiatry 2013; 202:115-20.

11. Grigoriadis S, VonderPorten EH, Mamisashvili L, Roerecke M, Rehm J, Dennis CL, et al. Antidepressant exposure during pregnancy and congenital malformations: is there an association? A systematic review and meta-analysis of the best evidence. J Clin Psychiatry 2013; 74(4):e293-308.

12. Guo L, Zhao D, Zhang R, Li S, Liu R, Wang H, et al. A Matched Case-Control Study on the Association Between Colds, Depressive Symptoms during Pregnancy and Congenital Heart Disease in Northwestern China. Sci Rep 2019; 9(1):589.

13. Ban L, Gibson JE, West J, Fiaschi L, Sokal R, Smeeth L, et al. Maternal depression, antidepressant prescriptions, and congenital anomaly risk in offspring: a population-based cohort study. Bjog 2014; 121(12):1471-81.

14. Kawamoto T, Nitta H, Murata K, Toda E, Tsukamoto N, Hasegawa M, et al. Rationale and study design of the Japan environment and children's study (JECS). BMC Public Health 2014; 14:25.

15. Michikawa T, Nitta H, Nakayama SF, Yamazaki S, Isobe T, Tamura K, et al. Baseline Profile of Participants in the Japan Environment and Children's Study (JECS). J Epidemiol 2018; 28(2):99-104.

16. Kessler RC, Andrews G, Colpe LJ, Hiripi E, Mroczek DK, Normand SL, et al. Short screening scales to monitor population prevalences and trends in nonspecific psychological distress. Psychol Med 2002; 32(6):959-76.

17. Furukawa TA, Kawakami N, Saitoh M, Ono Y, Nakane Y, Nakamura Y, et al. The performance of the Japanese version of the K6 and K10 in the World Mental Health Survey Japan. Int J Methods Psychiatr Res 2008; 17(3):152-8.

18. Prochaska JJ, Sung HY, Max W, Shi Y, Ong M. Validity study of the K6 scale as a measure of moderate mental distress based on mental health treatment need and utilization. Int J Methods Psychiatr Res 2012; 21(2):88-97.

19. Kessler RC, Barker PR, Colpe LJ, Epstein JF, Gfroerer JC, Hiripi E, et al. Screening for serious mental illness in the general population. Arch Gen Psychiatry 2003; 60(2):184-9.

20. Wurst KE, Poole C, Ephross SA, Olshan AF. First trimester paroxetine use and the prevalence of congenital, specifically cardiac, defects: a meta-analysis of epidemiological studies. Birth Defects Res A Clin Mol Teratol 2010; 88(3):159-70.

21. Myles $\mathrm{N}$, Newall H, Ward H, Large M. Systematic meta-analysis of individual selective serotonin reuptake inhibitor medications and congenital malformations. Aust N Z J Psychiatry 2013; 47(11):1002-12.

22. Huybrechts KF, Palmsten K, Avorn J, Cohen LS, Holmes LB, Franklin JM, et al. Antidepressant use in pregnancy and the risk of cardiac defects. N Engl J Med 2014; 370(25):2397-407.

23. Anderson KN, Lind JN, Simeone RM, Bobo WV, Mitchell AA, Riehle-Colarusso T, et al. Maternal Use of Specific Antidepressant Medications During Early Pregnancy and the Risk of Selected Birth Defects. JAMA Psychiatry 2020; 77(12):1246-55.

24. Murakami K, Miyake Y, Sasaki S, Tanaka K, Ohya Y, Hirota Y. Education, but not occupation or household income, is positively related to favorable dietary intake patterns in pregnant Japanese women: the Osaka Maternal and Child Health Study. Nutr Res 2009; 29(3):164-72.

25. Kagamimori S, Gaina A, Nasermoaddeli A. Socioeconomic status and health in the Japanese population. Soc Sci Med 2009; 68(12):2152-60. 
26. Kigawa M, Tsuchida A, Matsumura K, Takamori A, Ito M, Tanaka T, et al. Factors of non-responsive or lost-to-follow-up Japanese mothers during the first year post partum following the Japan Environment and Children's Study: a longitudinal cohort study. BMJ Open 2019; 9(11):e031222.

27. Rothman KJ, Greenland S, Lash TL: Modern Epidemiology: Lippincott Williams \& Wilkins; 2012.

28. Rychik J, Donaghue DD, Levy S, Fajardo C, Combs J, Zhang X, et al. Maternal psychological stress after prenatal diagnosis of congenital heart disease. J Pediatr 2013; 162(2):302-7.e1.

29. Satomi G. Guidelines for fetal echocardiography. Pediatr Int 2015; 57(1):1-21.

\section{Figures}

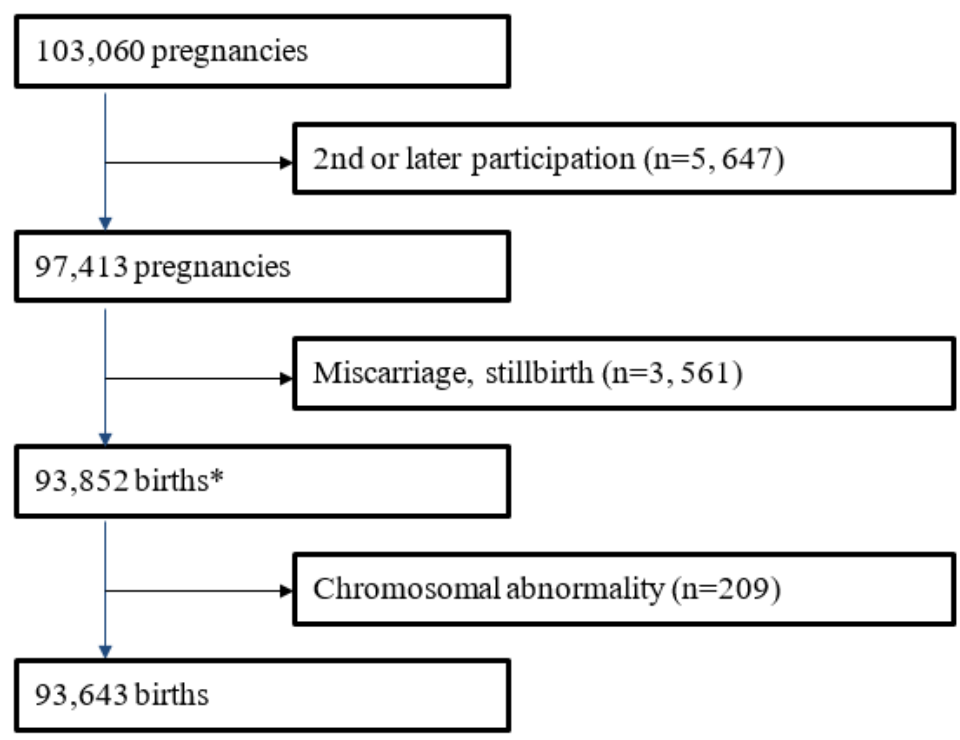

Figure 1

Flowchart of the study * Only the first infants of multiple births are included.

\section{Supplementary Files}

This is a list of supplementary files associated with this preprint. Click to download.

- Supplementaryfile.docx 